\title{
Dominance intensity measuring methods in MCDM with ordinal relations regarding weights
}

\author{
A. Mateos *, A. Jiménez-Martín, E.A. Aguayo, P. Sabio \\ Departamento de Inteligencia Artificial, Universidad Politécnica de Madrid, Campus de Montegancedo S/N, Boadilla del Monte, 28660 Madrid, Spain
}

A B S T R A C T

We consider a multicriteria decision-making context in which the decision-maker's preferences are represented by a multi-attribute additive value function. We account for imprecision concerning the per-formance of alternatives, value functions and weights, which represent the relative importance of criteria. We propose two new methods based on dominance intensity measures aimed at ranking alternatives. Both methods can be applied to different representations of imprecision about weights. Their perfor-mance is compared with other existing approaches when ordinal weight information represents impre-cision concerning weights. Monte Carlo simulation is used for the comparison in terms of a hit ratio and a rank-order correlation.

\section{Introduction}

A key concept in Multi-Attribute Value Theory (MAVT) refers to preferential independence conditions, see Keeney and Raiffa [7]. For reasons described in Raiffa [14] and Stewart [21], the additive model is considered a valid approach in many practical situations and is widely used. The functional form of the additive model is

$v\left(a_{i}\right)=\sum_{j=1}^{n} w_{j} v_{j}\left(x_{i j}\right)$,

where $x_{i j}$ is the performance over the attribute (or criterion) $X_{j}, j=1, \ldots, n$, for the alternative $a_{i}, i=1, \ldots, m$; and $v_{j}$ is the value function and $w_{j}$ is the weight, respectively, for attribute $X_{j}$. Note that $\sum_{j=1}^{n} w_{j}=1$ and $w_{j} \geqslant 0$.

However, it is often not easy to elicit precise value functions and/or values for scaling weights. They are often described within prescribed bounds or as just satisfying certain relations. Different authors refer to this situation as decision-making with imprecise information, incomplete information or partial information $[15,16]$.

Several reasons are given in the literature that justify why a decision maker (DM) may wish to provide imprecise information $[23,20]$. For instance, regarding alternative performances, some parameters of the model may be intangible as they reflect social

\footnotetext{
This paper was supported by the Madrid Regional Government project S-0505/ TIC/0230, the Spanish Ministry of Education and Science project TIN2008-06796C04-02 and the Spanish Ministry of Science and Innovation project MTM201128983-C03-03.

* Corresponding author. Tel.: +34 91336 6596; fax: +34913524819.

E-mail addresses: amateos@fi.upm.es (A. Mateos), ajimenez@fi.upm.es (A.Jiménez-Martín), ea.aguayo@upm.es (E.A. Aguayo), pilar.sabio@urjc.es (P.Sabio).
}

or environmental impacts. Also, performance may be taken from statistics or measurements, and the information that would set the value of some parameters may be incomplete, contradictory or controversial. Regarding weights, DMs may find it difficult to compare criteria or may not want to reveal their preferences in public. Moreover, the decision could be taken in a group decision-making situation, where imprecise information, such as weight rankings or weight intervals, is usually derived from a negotiation process $[6,11]$.

Many papers on MAVT have dealt with imprecise information. Sage and White [17] proposed the model of Imprecisely Specified Multi-Attribute Utility Theory (ISMAUT), where preference information about both weights and utilities is not assumed to be precise. Malakooti [10] suggested an efficient algorithm for ranking alternatives when there exists imprecise information about preferences and alternative values. Ahn [1] extended Malakooti's work. Lee et al. [9] extended the approach to hierarchical structures, and Park [12] developed the concepts of weak potential optimality and strong potential optimality. More recently, the TOPSIS method has been extended to uncertain linguistic environments $[25,26]$ or used for determining DM weights with interval numbers [27]. Dias [19] provide a brief overview of approaches proposed by different authors within the MAUT (Multi-Attribute Utility Theory) and MAVT framework to deal with imprecise information.

A recent approach to deal with imprecise information is computing different measures of dominance to derive a ranking of alternatives, known as dominance measuring methods. Ahn and Park [2] compute both dominating and dominated measures to derive net dominance. This is used as a measure of the strength of preference in the sense that a greater net value is better. However, the 
results of simulation experiments suggest that surrogate weighting methods, specifically the rank-order centroid weights (ROC) method, perform best in terms of selecting the best alternative and ranking alternatives.

In this paper we consider a decision-making problem with $n$ attributes, $X=\left\{X_{1}, \ldots, X_{n}\right\}$, and $m$ alternatives, $A=\left\{a_{1}, \ldots, a_{m}\right\}$, where $W\left(\mathbf{w}=\left(w_{1}, \ldots, w_{n}\right) \in W\right)$ and $V_{i}\left(\mathbf{v}_{i}=\left(v_{i}\left(x_{i 1}\right), \ldots, v_{i}\left(x_{i n}\right)\right)\right.$ $\in V_{i}$ ) define the feasible region for scaling weights and values associated with the alternative $a_{i}$ over each attribute, respectively, representing imprecise information. Therefore, Eq. (1) can be rewritten as

$$
v\left(a_{i}\right) \equiv\left\{\mathbf{w}^{T} \mathbf{v}_{i} \mid \mathbf{w} \in W, \mathbf{v}_{i} \in V_{i}\right\} .
$$

We analyze two new dominance measuring methods based on a dominance intensity that we will denote from now on as dominance intensity methods. The first method computes dominating and dominated measures in the manner of Ahn and Park [2], but these measures are combined into a dominance intensity rather than a net dominance measure. In the second method, a global dominance intensity measure is derived to rank alternatives. A simulation study is performed to compare the proposed methods with Ahn and Park [2] and with surrogate weighting methods and modified decision rules.

The paper is organized as follows. Section 2 reviews earlier methods for dealing with imprecise information within MCDM. Section 3 introduces two dominance intensity measuring methods. Section 4 illustrates the proposed methods with an example. Section 5 uses Monte Carlo simulation to compare the performance of the proposed methods with the approaches reviewed in Section 2. We outline the conclusions in Section 6.

\section{Review of earlier methods for dealing with imprecise information within MCDM}

Different approaches dealing with imprecise attribute weights to output a best alternative and/or ranking of alternatives can be found in the literature. In this section, we detail the approaches that will be compared with the methods that we propose in order to analyse their performance. We start with surrogate weighting methods, which can be used when there are ordinal relations regarding attribute weights. Next, we introduce some classical decision rules modified to account for imprecise decision contexts based on the absolute dominance concept. Finally, we describe two dominance measuring methods.

\subsection{Surrogate weighting methods}

If the DM provides ordinal relations regarding attribute weights, i.e., we consider a ranking of importance of attributes, arranged in descending order from the most to the least important attribute, then

$$
\begin{aligned}
\mathbf{w} & \in W \\
& =\left\{\mathbf{w}=\left(w_{1}, \ldots, w_{n}\right) \mid w_{1} \geqslant w_{2} \geqslant \cdots \geqslant w_{n} \geqslant 0, \sum_{i=1}^{n} w_{i}=1\right\} .
\end{aligned}
$$

Surrogate weighting methods deal with ranked attribute weights to output a best alternative and/or ranking of alternatives. In these methods, a weight vector is selected from a set of admissible weights to represent the set. This vector is then used to evaluate the alternatives by means of the multi-attribute value model. Commonly used surrogate weighting methods are rank sum (RS) weights and rank reciprocal (RR) weights, proposed in Stillwell et al. [22], or rank-order centroid (ROC) weights and equal (EW) weights, suggested by Barron and Barrett [3]. Table 1 shows how weights are computed for the above surrogate weighting methods.

The $R O C$ and $R R$ methods typically attach more importance to the best rankings. Compared with the ROC method, the $R R$ method distributes values more evenly over the worst rank. Thus, aggregation by the RR method is insensitive to the order of the worst ranks. On the other hand, the $E W$ method assigns equal weights to all the attributes, whereas the $R S$ method emphasizes all the weight rankings at the same level (i.e., $w_{1}-w_{2}=w_{2}-w_{3}=\cdots=w_{n-1}-w_{n}$ ).

Surrogate weighting methods have been evaluated by different authors (see, e.g., [4]). The common conclusion reached is that the $R O C$ method has an appealing theoretical rationale and appears to perform better than the other rank-based schemes in terms of choice accuracy. ROC has been extended to address the situation where alternative performances for an attribute are not precisely known, but again the DM has to provide ordinal information [20], i.e., a ranking of alternatives for the respective attributes.

\subsection{Dominance measuring methods}

A possibility described in the literature for dealing with imprecision is based on the concept of dominance. Given two alternatives $a_{k}$ and $a_{j}$, alternative $a_{k}$ dominates $a_{j}$ if $D_{k j} \geqslant 0$, with $D_{k j}$ being the optimum value of the optimization problem,

$D_{k j}=\min \left\{v\left(a_{k}\right)-v\left(a_{j}\right)=\mathbf{w}^{T} \mathbf{v}_{k}-\mathbf{w}^{T} \mathbf{v}_{j} \mid \mathbf{w} \in W, \mathbf{v}_{k} \in V_{k}, \mathbf{v}_{j} \in V_{j}\right\}$,

and there exists at least one $\mathbf{w}, \mathbf{v}_{k}$ and $\mathbf{v}_{j}$ such that the overall value of $a_{k}$ is strictly greater than that of $a_{j}$. This concept of dominance is called pairwise dominance.

Another type of dominance, known as absolute dominance [18], can be employed. Absolute dominance considers the following optimization problems:

$U_{k}=\max \left\{\mathbf{w}^{T} \mathbf{v}_{k} \mid \mathbf{w} \in W, \mathbf{v}_{k} \in V_{k}\right\}$ and

$L_{k}=\min \left\{\mathbf{w}^{T} \mathbf{v}_{k} \mid \mathbf{w} \in W, \mathbf{v}_{k} \in V_{k}\right\}$.

Alternative $a_{k}$ dominates absolutely $a_{j}$ if $L_{k} \geqslant U_{j}$. Note that if $a_{k}$ absolutely dominates $a_{j}$, then $a_{k}$ dominates $a_{j}$, but the reverse does not hold.

This dominance approach often results in almost no priorization of alternatives or too many non-dominated alternatives [8]. However, pairwise and absolute dominance values can be used to further prioritize competitive alternatives, and hence recommend the best alternative and fully rank alternatives. The use of these dominance values is exemplified by the modification of four classical decision rules to encompass an imprecise decision context $[13,18]$ :

- maximax or optimist rule (OPT) consists of evaluating each alternative based on its maximum guaranteed value, i.e., best case $\Rightarrow$ maximax: $\max _{j}\left\{U_{j}\right\}$.

- maximin or pessimist rule (PES) consists of evaluating each alternative based on its minimum guaranteed value, i.e., worst case $\Rightarrow$ maximin: $\max _{j}\left\{L_{j}\right\}$.

Table 1

Surrogate weighting methods.

\begin{tabular}{lll}
\hline Rank sum weights & $(R S)$ & $w_{i}=\frac{n+1-i}{\sum_{j-1}^{n} j}=\frac{2(n+1-i)}{n(n+1)}, i=1, \ldots, n$ \\
Rank reciprocal weights & $(R R)$ & $w_{i}=\frac{1 / i}{\sum_{j-1}^{n} 1 / j}, i=1, \ldots, n$ \\
Rank-order centroid weights & $(R O C)$ & $w_{i}=\frac{\sum_{j-i}^{n} 1 / j}{n}, i=1, \ldots, n$ \\
Equal weights & $(E W)$ & $w_{i}=\frac{1}{n}, i=1, \ldots, n$ \\
\hline
\end{tabular}


- minimax regret rule ( $R E G$ ) consists of evaluating each alternative based on the maximum loss of value with respect to a better alternative $\Rightarrow$ minimax regret: $\min _{k}\left\{M R_{k}\right\}$, where $M R_{k}$ represents the maximum regret incurred when choosing alternative $a_{k}$, i.e.,

$$
M R_{k}=\max \left\{\max \left\{\mathbf{w}^{T} \mathbf{v}_{j}-\mathbf{w}^{T} \mathbf{v}_{k} \mid \mathbf{w} \in W, \mathbf{v}_{j} \in V_{j}, \mathbf{v}_{k} \in V_{k}\right\} \forall j \neq k\right\} .
$$

- central value rule (CEN) consists of evaluating each alternative based on the midpoint of the range of possible performances $\Rightarrow$ central values: $\max _{k}\left\{\frac{U_{k}+L_{k}}{2}\right\}$.

Obviously none of these rules ensures that the best-ranked alternative coincides. However, simulations show that the selected alternative is generally one of the best [19].

A more recent approach is computing different measures of dominance to derive a ranking of alternatives, known as dominance measuring methods. For instance, Ahn and Park [2] compute a dominating measure $\phi_{k}^{+}=\sum_{\substack{j \neq 1 \\ j \neq k}}^{m} D_{k j}$ and a dominated measure $\phi_{k}^{-}=\sum_{\substack{j=1 \\ j \neq k}}^{m} D_{j k}$ for alternative $a_{k}$, and then derive a net dominance as $\phi_{k}=\phi_{k}^{+}-\phi_{k}^{-}$. With these measures, Ahn and Park proposed two methods:

- AP1: Rank alternatives according to $\phi_{k}^{+}$values, where the proposed alternative is that for which $\phi_{k}^{+}$is maximum.

- AP2: Rank alternatives according to $\phi_{k}$.

\section{Dominance intensity measuring methods}

We introduce two new dominance measuring methods based on dominance intensities, denoted dominance intensity measuring methods. The first one is based on the same idea as Ahn and Park [2]. It also computes dominating and dominated measures but they are combined into a dominance intensity rather than a net dominance index. In the second method, a global dominance intensity index is derived to rank alternatives. These are used as a measure of the strength of the preference in the sense that a greater value is better.

\subsection{Dominance intensity method 1 (DIM1)}

DIM1 is implemented as follows:

1. Obtain the paired dominance values $D_{k j}$ by solving problem Eq. (2) for $m(m-1)$ ordered pairs of alternatives, grouped in the matrix:

$$
D=\left(\begin{array}{ccccc}
- & D_{12} & \cdots & D_{1(m-1)} & D_{1 m} \\
D_{21} & - & \cdots & D_{2(m-1)} & D_{2 m} \\
D_{31} & D_{32} & \cdots & D_{3(m-1)} & D_{3 m} \\
\vdots & \vdots & \vdots & \vdots & \vdots \\
D_{m 1} & D_{m 2} & \cdots & D_{m(m-1)} & -
\end{array}\right) .
$$

2. Compute the dominating indices $\phi_{k}^{+}, \phi_{k}^{+>}$and $\phi_{k}^{+<}$for each alternative $a_{k}$ :

$$
\phi_{k}^{+}=\sum_{\substack{j=1 \\ j \neq k}}^{m} D_{k j}, \phi_{k}^{+>}=\sum_{\substack{j=1 \\ j \neq k \\ D_{k j}>0}}^{m} D_{k j}, \text { and } \phi_{k}^{+<}=\sum_{\substack{j-1 \\ j \neq k \\ D_{k j}<0}}^{m} D_{k j}, k=1, \ldots, m .
$$

In other words, $\phi_{k}^{+}$is computed adding the paired dominance values in the $k$ th row of $D$, whereas $\phi_{k}^{+>}$and $\phi_{k}^{+<}$are computed in the same way, but just considering positive and negative values in the corresponding row, respectively. Clearly, $\phi_{k}^{+}=\phi_{k}^{+>}+\phi_{k}^{+<}$.

\section{Compute the ratio}

$$
R_{k}^{+}=\frac{\phi_{k}^{+>}}{\phi_{k}^{+>}-\phi_{k}^{+<}}
$$

Note that $R_{k}^{+}$is well defined because we can assume that $0<\phi_{k}^{+>}-\phi_{k}^{+<}$, avoiding division by 0 , as demonstrated at the end of the algorithm. Note also that $0 \leqslant R_{k}^{+} \leqslant 1$.

4. Compute the dominated indices $\phi_{k}^{-}, \phi_{k}^{-<}$and $\phi_{k}^{->}$for each alternative $a_{k}$ :

$$
\phi_{k}^{-}=\sum_{\substack{j-1 \\ j \neq k}}^{m} D_{j k}, \phi_{k}^{->}=\sum_{\substack{j-1 \\ j \neq k \\ D_{j k}>0}}^{m} D_{j k}, \text { and } \phi_{k}^{-<}=\sum_{\substack{j=1 \\ j \neq k \\ D_{j k}<0}}^{m} D_{j k}, k=1, \ldots, m .
$$

In other words, $\phi_{k}^{-}$is computed by adding the paired dominance values in the $k$ th column of $D$, whereas $\phi_{k}^{->}$and $\phi_{k}^{-<}$are computed in the same way, but considering just the positive and negative values in the respective column. Note that $\phi_{k}^{-}=\phi_{k}^{->}+\phi_{k}^{-<}$.

5. Compute the ratio

$$
R_{k}^{-}=\frac{\phi_{k}^{->}}{\phi_{k}^{->}-\phi_{k}^{-<}}
$$

Note that $R_{k}^{-}$is also well defined because we can assume that $0<\phi_{k}^{->}-\phi_{k}^{-<}$, and $0 \leqslant R_{k}^{-} \leqslant 1$.

6. Calculate the global dominance intensity value $D_{k}$ for each alternative $a_{k}$ :

$$
D_{k}=R_{k}^{+}-R_{k}^{-}, \quad k=1, \ldots, m .
$$

Note that $-1 \leqslant D_{k} \leqslant 1$, where $-1=D_{k} \Longleftrightarrow\left(R_{k}^{+}=0\right.$ and $\left.R_{k}^{-}=1\right)$ when all alternatives dominate $a_{k}$, and $D_{k}=1 \Longleftrightarrow\left(R_{k}^{+}=1\right.$ and $R_{k}^{-}=0$ ) when all alternatives are dominated by $a_{k}$.

7. Rank alternatives according to $D_{k}$ values, where the best alternative is that for which $D_{k}$ is maximum and the worst is that for which $D_{k}$ is minimum.

Let us demonstrate that $R_{k}^{+}$is well defined. $R_{k}^{+}$would not be well defined if $\phi_{k}^{+>}-\phi_{k}^{+<}=0$. In this case,

$$
\begin{aligned}
& \phi_{k}^{+>}-\phi_{k}^{+<}=0 \Rightarrow\left\{\begin{array}{l}
\phi_{k}^{+>}=0 \\
\phi_{k}^{+<}=0
\end{array} \Rightarrow D_{k j}=0, \forall j\right. \\
& \Rightarrow \mathbf{w}^{T} \mathbf{v}_{k}-\mathbf{w}^{T} \mathbf{v}_{j} \geqslant 0, \forall j, \forall \mathbf{w} \Rightarrow \mathbf{w}^{T} \mathbf{v}_{j}-\mathbf{w}^{T} \mathbf{v}_{k} \leqslant 0, \forall j \Rightarrow D_{j k} \leqslant 0, \forall j .
\end{aligned}
$$

Therefore, we have two possibilities:

- $D_{k j}=0, \forall j$ and $D_{j k}<0, \forall j \Rightarrow$ alternative $a_{k}$ dominates $a_{j}, \forall j \Rightarrow a_{k}$ is the most preferred alternative. This may be recognized from the beginning, so we assume it does not happen.

- $D_{k j}=0, \forall j$ and $D_{j k}=0, \forall j \Rightarrow \mathbf{w}^{T} \mathbf{v}_{k}=\mathbf{w}^{T} \mathbf{v}_{j} \forall \mathbf{w} \in W \Rightarrow$ Both alternatives are indifferent. In this case, we can discard alternative $a_{j}$ and keep $a_{k}$ (or the opposite).

As a conclusion, if we assume that there are no two alternatives $a_{k}$ and $a_{j}$ with $\mathbf{w}^{T} \mathbf{v}_{k}=\mathbf{w}^{T} \mathbf{v}_{j} \forall \mathbf{w} \in W, \forall \mathbf{v}_{k} \in V_{k}, \forall \mathbf{v}_{j} \in V_{j}$, (in this case, alternative $a_{j}$, or alternative $a_{k}$, would be discarded because they are indifferent) and $\phi_{k}^{+>}-\phi_{k}^{+<}=0$, then alternative $a_{k}$ dominates $a_{j}$, i.e., alternative $a_{k}$ is preferred, and $a_{j}$ would be eliminated from the analysis.

The difference between this approach and the methods proposed by Ahn and Park is as follows. Ahn and Park's first method, $A P 1$, adds positive and negative values for the alternative in its respective row. This accounts for the dominance of this over the other alternatives. Likewise, Ahn and Park's second method, AP2, adds positive and negative values to both the row and column. The two quantities are then subtracted. The values added in the column account for the dominance of the other alternatives over 
the alternative in question. As pointed out in Section 2, a simulation study showed $A P 1$ to be better than $A P 2$. The reason is that $A P 2$ uses duplicate information (row and column values).

The starting point for DIM1 is AP2. The aim is to improve AP2 by reducing the duplicate information involved in the computations. We have taken into account that a given alternative, $a_{k}$, only dominates alternatives with positive elements in the $k$ th row of the dominance matrix. Analogously, this alternative is dominated only by alternatives with positive elements in the $k$ th column.

Therefore, we compute the dominating measure by adding positive values in the respective row, which we divide by the difference between the sums of the positive and negative values in that row. Likewise, we compute the dominated measure by adding positive values in the respective column, which we divide by the difference between the sums of the positive and negative values in that column.

This type of ratios attach more weight to positive values in the row (or column). This means that DIM1 would output a value greater than zero in the event of positive values being canceled out by negative values where the dominating (dominated) measures in $A P 1$ and $A P 2$ would be zero.

However, DIM 1 has a drawback: if all the elements in $D$ are negative then it is not possible to derive a ranking of alternatives because their dominance intensity is zero in all cases, since $\phi_{k}^{+>}=0$ and $\phi_{k}^{->}=0, \forall k$. This drawback implies that DIM1 is not independent of irrelevant alternatives. A simple numerical example follows. Consider a two-criteria problem. The only constraints on weights are that they should be non-negative and add up to 1 . Alternatives are $a_{1} \equiv(e, 1+e), a_{2} \equiv(0,1)$ and $a_{3} \equiv(1,0)$ ( $e$ is a number between 0 and 1$)$. Considering all three alternatives, $a_{1}$ is the best-ranked according to DIM1. But this is because we have included $a_{2}$, which is irrelevant. If we remove $a_{2}$, DIM1 is unable to rank the alternatives. In Section 3.2, we propose another dominance intensity method that overcomes this drawback.

\subsection{Dominance intensity method 2 (DIM2)}

To introduce the second dominance intensity method, DIM2, we observe that, trivially,

$D_{k j} \leqslant \mathbf{w}^{T}\left(\mathbf{v}_{k}-\mathbf{v}_{j}\right) \leqslant-D_{j k}, \forall \mathbf{w} \in W, \mathbf{v}_{k} \in V_{k}, \mathbf{v}_{j} \in V_{j}$.

In this method, paired dominance values $D_{k j}$ are first transformed into dominance intensities $D I_{k j}$. Then, a global dominance intensity $\left(G D I_{k}\right)$ is derived for each alternative $a_{k}$. This is used as a measure of the strength of preference, in the sense that a greater global dominance intensity is better.

DIM2 is implemented as follows:

1. Obtain matrix $D$ as before.

2. If $D_{k j} \geqslant 0$, then alternative $a_{k}$ dominates alternative $a_{j}$, and we say that the dominance intensity of $a_{k}$ over $a_{j}$ is 1 , i.e., $D I_{k j}=1$. Else $\left(D_{k j}<0\right)$ :

- If $D_{j k} \geqslant 0$, then alternative $a_{j}$ dominates alternative $a_{k}$, and we say that the dominance intensity of $a_{k}$ over $a_{j}$ is 0 , i.e., $D I_{k j}=0$.

- Else, $\left(D_{j k}<0\right)$ the dominance intensity of $a_{k}$ over $a_{j}$ is defined as

$$
\text { as } I_{k j}=\frac{-D_{j k}}{-D_{j k}-D_{k j}} .
$$

3. Calculate a global dominance intensity (GDI) for each alternative $a_{k}$

$$
G D I_{k}=\sum_{j=1, j \neq k}^{m} D I_{k j}
$$

4. Rank alternatives according to the $G D I_{k}$ values, where the best (rank 1) is the alternative for which $G D I_{k}$ is maximum and the worst is the alternative for which $G D I_{k}$ is minimum.

In Section 5, we analyse the performance of the proposed methods and compare them with other methods reviewed in Section 2.

\section{A numerical example}

In this section, we provide a simple example to illustrate the proposed dominance intensity measuring methods. Let us consider a decision-making problem with five attributes, $X_{i}, i=1, \ldots, 5$ and five alternatives, $a_{j}, j=1, \ldots, 5$. We assume that the ranking of attribute importance is $1 \geqslant w_{1} \geqslant w_{2} \geqslant w_{3} \geqslant w_{4} \geqslant w_{5} \geqslant 0$, with $\sum_{i=1}^{5} w_{i}=1$

\begin{tabular}{|c|c|c|c|c|}
\hline$X_{1}$ & $X_{2}$ & $X_{3}$ & $X_{4}$ & $X_{E}$ \\
\hline 0.711 & 0.146 & 0.115 & 0.892 & 0.2 \\
\hline 0.253 & 0.192 & 0.087 & 0.722 & 0.477 \\
\hline 0.401 & 0.805 & 0.524 & 0.327 & 0.828 \\
\hline 0.793 & 0.965 & 0.647 & 0.241 & 0.091 \\
\hline 0.284 & 0.576 & 0.714 & 0.618 & 0.411 \\
\hline
\end{tabular}

The evaluation of the five alternatives is as follows

For convenience, the columns in this matrix are normalized so that the smallest value is zero and the largest value is one:

$\left(\begin{array}{ccccc}0.848 & 0 & 0.044 & 1 & 0.203 \\ 0 & 0.056 & 0 & 0.739 & 0.523 \\ 0.4274 & 0.804 & 0.697 & 0.132 & 1 \\ 1 & 1 & 0.893 & 0 & 0 \\ 0.057 & 0.525 & 0.714 & 1 & 0.434\end{array}\right)$.

Optimization problems are solved to compute paired dominance values, leading to the corresponding dominance matrix:

$D I=\begin{aligned} & a_{1} \\ & a_{2} \\ & a_{3} \\ & a_{4} \\ & a_{5}\end{aligned}\left(\begin{array}{ccccc}a_{1} & a_{2} & a_{3} & a_{4} & a_{5} \\ - & 1 & 0.66 & 0 & 0.77 \\ 0.34 & - & 0 & 0 & 0 \\ 1 & 1 & - & 0,004 & 0.80 \\ 0.22 & 1 & 0.99 & - & 1 \\ 0.20 & 0 & -\end{array}\right)$.

In the DIM1 method, the dominating measures, $\phi_{k}^{+>}$and $\phi_{k}^{+<}$, are computed for each alternative, followed by the ratios $R_{k}^{+}$, see Table 2. For instance, $\phi_{4}^{+>}=0.152+0.3148+0.0596=0.5264$ or $\phi_{3}^{+<}=-0.574-0.726-0.0633=-1.3633$. Next, the dominated measures $\phi_{k}^{-<}$and $\phi_{k}^{->}$are computed for each alternative, followed by the proportion $R_{k}^{-}$, shown in Table 2 .

Finally, the ranking of alternatives is derived from the global dominance intensity $D_{k}$, see Table 3 .

In the DIM2 dominance intensity measuring method, once the paired dominance matrix $D$ is available, dominance intensities $D I_{k j}$

\begin{tabular}{|c|c|c|c|c|c|c|}
\hline \multirow{6}{*}{$D I=$} & & $a_{1}$ & $a_{2}$ & $a_{3}$ & $a_{4}$ & $a_{5}$ \\
\hline & $a_{1}$ & - & 1 & 0.66 & 0 & 0.77 \\
\hline & $a_{2}$ & 0 & - & 0 & 0 & 0 \\
\hline & $a_{3}$ & 0.34 & 1 & - & 0,004 & 0.80 \\
\hline & $a_{4}$ & 1 & 1 & 0.99 & - & 1 \\
\hline & $a_{5}$ & 0.22 & 1 & 0.20 & 0 & - \\
\hline
\end{tabular}
are computed, leading to the matrix:

Table 2

\begin{tabular}{|c|c|c|c|c|c|c|c|}
\hline \multicolumn{4}{|c|}{ Dominating measures } & \multicolumn{4}{|c|}{ Dominated measures } \\
\hline Altern. & $\phi_{k}^{+>}$ & $\phi_{k}^{+<}$ & $R_{k}^{+}$ & Altern. & $\phi_{k}^{->}$ & $\phi_{k}^{-<}$ & $R_{\dot{k}}^{-}$ \\
\hline$a_{1}$ & 0.155 & -1.1907 & 0.11518 & $a_{1}$ & 0.152 & -2.2131 & 0.0643 \\
\hline$a_{2}$ & 0 & -2.93 & 0 & $a_{2}$ & 0.8008 & 0 & 1 \\
\hline$a_{3}$ & 0.274 & -1.3633 & 0.16734 & $a_{3}$ & 0 & -1.1191 & 0 \\
\hline$a_{4}$ & 0.5264 & -0.003 & 0.99433 & $a_{4}$ & 0 & -3.3357 & 0 \\
\hline$a_{5}$ & 0.057 & -1.9826 & 0.02794 & $a_{5}$ & 0.0596 & -0.8017 & 0.0692 \\
\hline
\end{tabular}

Dominating and dominated measures. 
Table 3

Global dominance intensities and ranking with DIM1.

\begin{tabular}{llllll}
\hline & $a_{1}$ & $a_{2}$ & $a_{3}$ & $a_{4}$ & $a_{5}$ \\
\hline$D_{k}$ & 0.05 & -1 & 0.17 & 0.99 & -0.04 \\
Ranking & 3 & 5 & 2 & 1 & 4 \\
\hline
\end{tabular}

Table 4

Global dominance intensities and ranking with DIM2.

\begin{tabular}{llllll}
\hline & $a_{1}$ & $a_{2}$ & $a_{3}$ & $a_{4}$ & $a_{5}$ \\
\hline$G D I_{k}$ & 2.44 & 0.00 & 2.14 & 3.99 & 1.43 \\
Ranking & 2 & 5 & 3 & 1 & 4 \\
\hline
\end{tabular}

For instance, as $D_{12}=0.155 \geqslant 0$, then $a_{1}$ dominates $a_{2}$ and $D I_{12}=1$. On the other hand, $D_{14}=-0.6667<0$, whereas $D_{41}=0.152>0$, so $D I_{14}=0$.

Finally, $\quad D_{13}=-0.2943<0$ and $D_{31}=-0.574<0$. Consequently, $D I_{13}=\frac{0.574}{0.2943+0.574}=0.66$.

Finally, the ranking of alternatives is derived from the global dominance intensity $G D I_{k}$ (see Table 4).

We find that the rankings output by both methods (DIM1 and DIM2), see Tables 3 and 4 , are very similar but do not match exactly. The best- and worst-ranked alternatives are the same for both methods ( $a_{4}$, and $a_{5}$ and $a_{2}$, respectively). However, the alternatives ranked second and third are different for each method $\left(a_{1}\right.$ and $a_{3}$, respectively). The question is, then, does one method outperform the other for different decision-making settings, i.e., for a different number of attributes or alternatives?

\section{Performance analysis based on Monte Carlo simulation}

Having described the dominance intensity measuring methods we propose ( DIM1 and DIM2), let us now compare these methods with Ahn and Park's approach, surrogate weighting methods and decision rules modified to encompass an imprecise decision-making context. Note that, although DIM1 could be discarded from further analysis since it is not independent of irrelevant alternatives, as explained in Section 3.1, it has been considered in the simulation process.

The proposed methods can be applied for different representations of imprecision concerning weights. In surrogate weighting methods, however, DMs provide ordinal relations regarding attribute weights. For comparability, we have considered ordinal relations about weights throughout the simulation.

We shall carry out a simulation study of the above methods to analyze their performance. For a decision-making problem with $m$ alternatives and $n$ attributes, the process would be as follows:

1. Component utilities are randomly generated for each alternative in each attribute from a uniform distribution in $(0,1)$, leading to an $m \times n$ matrix. The columns in this matrix are normalized to make the smallest value zero and the largest value one, and dominated alternatives are removed. Note that these alternatives are removed in the simulation because they are not useful for analyzing the performance of the considered methods.

2. Attribute weights are randomly generated according to the ranking of attribute importance [5]. If these weights are the TRUE weights, the derived ranking of alternatives will be denoted as the TRUE ranking. To generate the TRUE weights, first select $n-1$ independent random numbers from a uniform distribution on $(0,1)$, and rank these numbers. Suppose the ranked numbers are $1 \geqslant r_{n-1} \geqslant \cdots \geqslant r_{2} \geqslant r_{1}>0$. The differences between adjacently ranked numbers are then used as the target weights $\quad w_{n}^{T}=1-r_{n-1}, w_{n-1}^{T}=r_{n-1}-r_{n-2}, \ldots, w_{1}^{T}=r_{1} . \quad$ The resulting weights will sum 1 and be uniformly distributed in the weight space. Attribute weights corresponding to surrogate weighting methods are computed as described in the introduction.

3. The ranking of alternatives is computed for each method (surrogate weighting methods, modified decision rules and dominance measuring methods) according to their procedures and compared with the TRUE ranking, computed in the last step. We use two measures of efficacy, the hit ratio and rank-order correlation $[3,2]$. The hit ratio is the proportion of cases in which the method selects the same best alternative as in the TRUE ranking. Rank-order correlation represents how similar the overall rank structures of alternatives are in the TRUE ranking and in the ranking derived from the method. It is calculated using Kendall's $\tau$ [24]:

$$
\begin{aligned}
\tau & =1-\frac{2 \times(\text { number of pairwise preference violations })}{\text { Total number of pair preferences }} \\
& =\frac{S}{m(m-1) / 2},
\end{aligned}
$$

where $S$ is the difference between the number of concordant (ordered in the same way) and discordant (ordered differently) pairs and $m$ is the total number of alternatives.

If there are tied (same value) observations then the denominator $m(m-1) / 2$ has to be replaced by

$$
\sqrt{\left[m(m-1) / 2-\sum_{i 1}^{t} t_{i}\left(t_{i}-1\right) / 2\right]\left[m(m-1) / 2-\sum_{i 1}^{t} u_{i}\left(u_{i}-1\right) / 2\right]},
$$

where $t_{i}$ is the number of observations tied at the TRUE ranking, and $u_{i}$ is the number of observations tied at the ranking derived from the method.

Like Ahn and Park [2], we validated the results using four different levels of alternatives $(m=3,5,7,10)$ and five different levels of attributes $(n=3,5,7,10,15)$. Also, 10 replications of 10,000 trials were performed for each of the 20 design elements (alternatives $x$ attributes). Replications were parallelized to save computational resources (mainly time).

Table 5 exhibits the average hit ratio for each of the 20 design elements, i.e., the average values of 10 replications of 10,000 trials, whereas the last row in this table is the mean of each column.

Looking at the surrogate weighting methods, $R O C$ is the best, followed by the $R R$ method, the $R S$ method and the $E W$ method. This result matches those reported in Barron and Barrett [3] and Ahn and Park [2]. Regarding modified decision rules, although there is no regular trend, the PES method appears to be better than the CEN method. CEN outperforms the REG method, and REG outperforms the OPTmethod. In any case, modified decision rules are worse than surrogate weighting methods, except for the EW method. For the dominance intensity methods, the mean value for the DIM2 method is the highest ( 81.8 ), its hit ratio being the highest for 13 out of the 20 design elements. The DIM2 method is followed by DIM1, which is better than AP1. AP1 is better than AP2.

On the whole, ROC and DIM2 are better than the other methods in terms of hit ratio. The DIM2 method performs better than the dominance measuring methods in Ahn and Park [2], and outputs results closer to the ROC method. Furthermore, according to the paired-samples $t$-test (which computes the difference between the mean values of the two methods and tests whether the average differs from zero), there is no significant difference among the hit ratio means for the DIM2 and ROC methods (significance level, two-tailed: 0.210 ).

$R O C$ is again the best surrogate weighting method in terms of rank-order correlation (see Table 6), followed by $R R, R S$ and $E W$. 
Table 5

Average hit ratios.

\begin{tabular}{|c|c|c|c|c|c|c|c|c|c|}
\hline \multirow[t]{2}{*}{ Alt. } & \multirow[t]{2}{*}{ Crit. } & \multicolumn{4}{|c|}{ Surrogate weighting } & \multicolumn{4}{|c|}{ Modified decision rules } \\
\hline & & $R S$ & $R R$ & ROC & $E W$ & $O P T$ & PES & $R E G$ & CEN \\
\hline \multirow[t]{5}{*}{3} & 3 & 89.6 & 92.5 & 92.5 & 89.6 & 77.1 & 89.6 & 92.5 & 92.5 \\
\hline & 5 & 82.5 & 88.0 & 88.0 & 81.2 & 74.8 & 81.4 & 84.8 & 84.8 \\
\hline & 7 & 79.4 & 78.4 & 79.9 & 73.4 & 56.0 & 73.5 & 65.9 & 65.9 \\
\hline & 10 & 94.8 & 94.8 & 94.8 & 84.8 & 59.1 & 94.8 & 75.1 & 79.0 \\
\hline & 15 & 78.2 & 87.8 & 90.1 & 57.1 & 53.8 & 71.7 & 69.4 & 69.4 \\
\hline \multirow[t]{5}{*}{5} & 3 & 82.4 & 83.1 & 84.4 & 53.9 & 46.6 & 65.0 & 84.4 & 83.1 \\
\hline & 5 & 93.5 & 93.5 & 93.5 & 38.3 & 62.2 & 83.8 & 93.5 & 93.5 \\
\hline & 7 & 90.9 & 90.9 & 90.9 & 71.2 & 44.7 & 84.8 & 70.2 & 64.7 \\
\hline & 10 & 78.3 & 64.3 & 79.2 & 59.9 & 29.5 & 56.7 & 58.4 & 56.1 \\
\hline & 15 & 89.3 & 85.1 & 91.1 & 59.5 & 46.9 & 81.3 & 58.9 & 66.9 \\
\hline \multirow[t]{5}{*}{7} & 3 & 63.0 & 65.7 & 66.6 & 45.1 & 36.1 & 60.8 & 64.9 & 62.5 \\
\hline & 5 & 68.8 & 71.4 & 77.2 & 47.9 & 42.6 & 71.4 & 61.8 & 64.1 \\
\hline & 7 & 84.6 & 84.6 & 84.6 & 43.5 & 39.8 & 72.1 & 66.3 & 49.0 \\
\hline & 10 & 64.7 & 64.2 & 72.3 & 48.7 & 40.8 & 51.7 & 49.2 & 50.4 \\
\hline & 15 & 71.7 & 78.5 & 87.8 & 50.0 & 51.5 & 80.2 & 53.2 & 61.5 \\
\hline \multirow[t]{5}{*}{10} & 3 & 73.4 & 73.4 & 73.4 & 42.2 & 37.9 & 53.0 & 65.0 & 73.4 \\
\hline & 5 & 72.2 & 66.2 & 72.0 & 30.4 & 42.1 & 55.4 & 66.4 & 66.3 \\
\hline & 7 & 72.9 & 80.6 & 80.6 & 32.6 & 31.4 & 80.6 & 67.7 & 80.6 \\
\hline & 10 & 76.7 & 76.7 & 88.3 & 58.4 & 14.0 & 62.2 & 47.1 & 32.9 \\
\hline & 15 & 80.7 & 82.4 & 85.7 & 78.0 & 22.2 & 81.2 & 60.4 & 61.5 \\
\hline Mean & & 79.94 & 80.1 & 83.6 & 57.3 & 45.6 & 72.5 & 67.7 & 67.9 \\
\hline \multirow[t]{2}{*}{ Alt. } & & \multirow[t]{2}{*}{ Crit. } & \multicolumn{7}{|c|}{ Dominance measuring } \\
\hline & & & $A P 1$ & & $A P 2$ & & DIM1 & & DIM2 \\
\hline \multirow[t]{5}{*}{3} & & 3 & 92.5 & & 92.5 & & 89.6 & & 94.6 \\
\hline & & 5 & 84.8 & & 84.8 & & 94.2 & & 93.3 \\
\hline & & 7 & 74.4 & & 65.9 & & 95.3 & & 77.4 \\
\hline & & 10 & 88.7 & & 79.0 & & 96.7 & & 98.9 \\
\hline & & 15 & 78.6 & & 69.4 & & 84.8 & & 78.6 \\
\hline \multirow[t]{5}{*}{5} & & 3 & 82.4 & & 84.4 & & 76.8 & & 82.4 \\
\hline & & 5 & 93.5 & & 93.5 & & 72.9 & & 93.5 \\
\hline & & 7 & 88.4 & & 64.7 & & 88.4 & & 88.4 \\
\hline & & 10 & 73.0 & & 41.8 & & 85.3 & & 82.5 \\
\hline & & 15 & 82.7 & & 66.9 & & 95.7 & & 80.4 \\
\hline \multirow[t]{5}{*}{7} & & 3 & 65.7 & & 66.5 & & 64.0 & & 73.1 \\
\hline & & 5 & 67.4 & & 68.7 & & 73.0 & & 74.6 \\
\hline & & 7 & 80.1 & & 66.3 & & 70.3 & & 76.0 \\
\hline & & 10 & 62.2 & & 61.1 & & 57.8 & & 66.3 \\
\hline & & 15 & 78.5 & & 61.5 & & 56.5 & & 78.5 \\
\hline \multirow[t]{5}{*}{10} & & 3 & 69.6 & & 73.4 & & 70.4 & & 86.0 \\
\hline & & 5 & 68.1 & & 66.2 & & 68.2 & & 70.2 \\
\hline & & 7 & 76.7 & & 71.5 & & 72.7 & & 79.3 \\
\hline & & 10 & 73.2 & & 48.0 & & 81.2 & & 76.7 \\
\hline & & 15 & 80.0 & & 73.7 & & 80.0 & & 84.6 \\
\hline Mean & & & 78.0 & & 70.0 & & 78.7 & & 81.8 \\
\hline
\end{tabular}

In the $R O C$ method, the rank-order correlations range from $71.6 \%$ to $90.7 \%$, and the values are relatively constant irrespective of the number of alternatives (mean values are $84.2 \%, 86 \%, 84.6 \%$ and $85 \%$ for $3,5,7$ and 10 alternatives, respectively). With exception of $E W$, modified decision rules are worse than the surrogate weighting method.

As regards dominance intensity measuring methods, DIM2 performs better than DIM1 and the approaches suggested by Ahn and Park [2], and reaches a rank-order correlation close to the ROC method. Its rank-order correlations range from $66.2 \%$ to $87.8 \%$, and are relatively constant irrespective of the number of alternatives (mean values are $82.6 \%, 77.1 \%, 80.4 \%$ and $80.6 \%$, respectively). In this case, the paired-samples t-test shows a significant difference among the rank-order correlation means for the DIM2 and ROC methods (significance level, two-tailed: 0.009 ). On the other hand,
Table 6

Rank-order correlation (Kendall's $\tau$ ).

\begin{tabular}{|c|c|c|c|c|c|c|c|c|c|}
\hline \multirow[t]{2}{*}{ Alt. } & \multirow[t]{2}{*}{ Crit. } & \multicolumn{4}{|c|}{ Surrogate weighting } & \multicolumn{4}{|c|}{ Modified decision rules } \\
\hline & & RS & $R R$ & ROC & $E W$ & $O P T$ & PES & $R E G$ & CEN \\
\hline \multirow[t]{5}{*}{3} & 3 & 85.5 & 88.9 & 88.9 & 60.6 & 66.7 & 87.0 & 88.9 & 88.9 \\
\hline & 5 & 86.4 & 89.9 & 89.9 & 62.8 & 75.8 & 70.7 & 77.0 & 82.5 \\
\hline & 7 & 64.4 & 65.5 & 71.6 & 59.4 & 49.7 & 52.7 & 45.9 & 45.9 \\
\hline & 10 & 79.3 & 86.2 & 88.1 & 66.8 & 60.3 & 79.5 & 66.4 & 73.6 \\
\hline & 15 & 63.5 & 74.7 & 82.6 & 27.1 & 55.5 & 57.3 & 55.8 & 62.5 \\
\hline \multirow[t]{5}{*}{5} & 3 & 81.8 & 84.0 & 84.5 & 46.5 & 62.6 & 77.9 & 80.1 & 82.3 \\
\hline & 5 & 84.2 & 85.8 & 86.4 & 45.4 & 73.5 & 75.1 & 73.7 & 80.1 \\
\hline & 7 & 83.0 & 84.7 & 87.8 & 62.3 & 68.2 & 70.8 & 65.5 & 73.8 \\
\hline & 10 & 78.1 & 76.1 & 83.1 & 54.2 & 65.3 & 56.1 & 73.7 & 64.9 \\
\hline & 15 & 83.8 & 78.6 & 88.5 & 58.6 & 58.4 & 54.4 & 49.9 & 58.6 \\
\hline \multirow[t]{5}{*}{7} & 3 & 78.6 & 80.6 & 81.5 & 48.3 & 71.0 & 74.6 & 73.5 & 78.7 \\
\hline & 5 & 77.0 & 80.1 & 82.0 & 47.4 & 70.4 & 70.9 & 66.2 & 74.5 \\
\hline & 7 & 81.3 & 85.7 & 87.3 & 49.3 & 70.6 & 70.3 & 68.0 & 73.6 \\
\hline & 10 & 75.9 & 77.1 & 84.4 & 54.6 & 58.7 & 58.6 & 52.9 & 62.8 \\
\hline & 15 & 79.3 & 79.0 & 87.9 & 55.2 & 70.7 & 64.0 & 56.2 & 66.6 \\
\hline \multirow[t]{5}{*}{10} & 3 & 75.4 & 77.0 & 79.6 & 36.6 & 57.8 & 72.3 & 74.9 & 77.0 \\
\hline & 5 & 77.6 & 81.1 & 82.9 & 51.3 & 66.3 & 68.9 & 62.4 & 75.1 \\
\hline & 7 & 78.0 & 83.1 & 85.2 & 50.0 & 69.8 & 69.2 & 60.0 & 75.0 \\
\hline & 10 & 78.9 & 81.2 & 87.0 & 53.3 & 69.8 & 58.1 & 48.8 & 65.3 \\
\hline & 15 & 81.3 & 85.6 & 90.7 & 53.6 & 67.7 & 70.1 & 62.3 & 72.3 \\
\hline Mean & & 78.6 & 81.2 & 85.0 & 52.1 & 65.4 & 67.9 & 64.1 & 71.7 \\
\hline \multirow[t]{2}{*}{ Alt. } & & \multirow[t]{2}{*}{ Crit. } & \multicolumn{7}{|c|}{ Dominance measuring } \\
\hline & & & & & $A P 2$ & & \multicolumn{2}{|l|}{ DIM1 } & DIM2 \\
\hline \multirow[t]{5}{*}{3} & & 3 & \multicolumn{2}{|c|}{88.9} & 88.9 & & 70.9 & & 87.5 \\
\hline & & 5 & & & 87.8 & & 77.1 & & 87.8 \\
\hline & & 7 & & & 51.2 & & 68.9 & & 83.4 \\
\hline & & 10 & & & 75.7 & & 59.3 & & 85.6 \\
\hline & & 15 & & & 62.5 & & 29.9 & & 68.6 \\
\hline \multirow[t]{5}{*}{5} & \multicolumn{2}{|r|}{3} & \multicolumn{2}{|c|}{83.5} & \multicolumn{2}{|l|}{83.3} & \multicolumn{2}{|l|}{76.8} & 82.5 \\
\hline & & 5 & & & 82.6 & & 78.2 & & 83.7 \\
\hline & & 7 & & & 74.2 & & 69.5 & & 82.3 \\
\hline & & 10 & & & 62.1 & & 49.1 & & 70.9 \\
\hline & & 15 & & & 56.2 & & 53.1 & & 66.2 \\
\hline 7 & & 3 & & & 79.2 & & 76.9 & & 80.3 \\
\hline & & 5 & & & 76.1 & & 78.7 & & 82.2 \\
\hline & & 7 & & & 79.2 & & 82.4 & & 82.8 \\
\hline & & 10 & & & 66.1 & & 85.3 & & 83.5 \\
\hline & & 15 & & & 67.3 & & 63.1 & & 73.4 \\
\hline 10 & & 3 & & & 78.4 & & 80.5 & & 81.7 \\
\hline & & 5 & & & 76.8 & & 83.4 & & 82.7 \\
\hline & & 7 & & & 76.8 & & 84.0 & & 82.3 \\
\hline & & 10 & & & 68.4 & & 78.7 & & 75.7 \\
\hline & & 15 & & & 77.5 & & 81.4 & & 80.7 \\
\hline Mean & & & & & 73.5 & & 71.4 & & 80.2 \\
\hline
\end{tabular}

DIM1 has the worst rank-order correlation mean out of all the dominance intensity measuring methods, this value improves in proportion to the number of alternatives ranked (mean values for $3,5,7$ and 10 alternatives are $61.2 \%, 65.3 \%, 77.3 \%$ and $81.6 \%$, respectively), see Table 6 .

Note that $R O C$ is an example of centroid values, which generalizes to any convex value set defined by linear inequalities, and centroid computations are relatively straightforward for a large class of situations. However, we think that DIM2 is more generally applicable in the sense that, it is easy to apply even to a non-convex value set (corresponding to different types of imprecision concerning the input parameters), once the respective optimization problems have been solved (possibly based on the application of metaheuristics) and the dominance matrix has been obtained. 


\section{Conclusions}

In complex decision-making problems it is often not easy to elicit precise values for scaling weights representing the relative importance of criteria, which are described by a constraint set, for instance, within prescribed bounds or as just satisfying certain ordinal relations (ranked attribute weights).

Two distinct approaches for suggesting an alternative and/or ranking of alternatives can be found in the literature dealing with ranked attribute weights. One group is the so-called surrogate weighting methods, where a weight vector is selected from a set of admissible weights to represent that set. This vector is then used to evaluate the alternatives by means of a multi-attribute value model. The other possibility is to eliminate inferior alternatives based on the concept of dominance. An example of the employment of dominance values is the modification of classical decision rules to encompass an imprecise decision context. A more recent approach is to use information about each alternative's intensity of dominance, known as dominance intensity measuring methods.

We have proposed two new dominance intensity measuring methods, DIM1 and DIM2. DIM1 is designed to improve one of the methods proposed by Ahn and Park, AP2, by reducing duplicate information to compute dominating and dominated measures. These measures are then combined to yield a dominance intensity. However, DIM1 was dispensed with because it is not independent of irrelevant alternatives. DIM2 derives a global dominance intensity measure to rank alternatives.

Monte Carlo simulation techniques have been used to analyse the performance of the proposed methods and to compare them with dominance measuring methods proposed by other authors (Ahn and Park), surrogate weighting methods and adapted decision rules.

As regards average hit ratios, DIM2 and ROC outperform the other methods (including DIM1) and, according to the paired-samples $t$-test, there is no significant difference among them. DIM2 and ROC also outperform the other methods (including DIM1) in terms of rank-order correlation, but ROC slighty outperforms DIM2 in this case.

Note, however, that $R O C$ can be only applied when ordinal relations regarding attribute weights are provided. However, DIM2 is more generally applicable since it can also be used when the imprecision concerning weights or even value functions is represented in other ways, for example by interval values, probability distributions or even fuzzy numbers. And again it is applicable when there is uncertainty about the alternative performances.

\section{References}

[1] B.S. Ahn, Extending Malakooti's model for ranking multicriteria alternatives with preference strength and partial information, IEEE Trans. Syst., Man. Cyb: Part A 33 (2003) 281-287.

[2] B.S. Ahn, K.S. Park, Comparing methods for multiattribute decision making with ordinal weights, Comput. Oper. Res. 35 (2008) 1660-1670.
[3] F. Barron, B. Barrett, Decision quality using ranked attribute weights, Man. Sci. 42 (1996) 1515-1523.

[4] J. Butler, D.L. Olson, Comparison of centroid and simulation approaches for selecting sensitivity analysis, J. Multi-Crit. Decis. Anal. 8 (1999) 146-161.

[5] J. Jia, G.W. Fischer, J.S. Dyer, Attribute weighting method and decision quality in the presence of response error: A simulation study, J. Behav. Decis. Making 11 (1998) 85-105.

[6] A. Jiménez, A. Mateos, S. Ríos Insua, Monte Carlo simulation techniques in a decision support system for group decision-making, Group Dec. Negot. 14 (2005) 109-130.

[7] R.L. Keeney, H. Raiffa, Decision with Multiple Objectives: Preferences and Value-Tradeoffs, Wiley, New York, 1976.

[8] C.W. Kirkwood, J.L. Corner, The effectiveness of partial information about attribute weights for ranking alternatives in multiattribute decision making, Org. Behav. Human Dec. Proc. 54 (1993) 456-476.

[9] K. Lee, K.S. Park, H. Kim, Dominance, potential optimality, imprecise information and hierarchical structure in multi-criteria analysis, Comput. Oper. Res. 29 (2002) 1267-1281.

[10] B. Malakooti, Ranking and screening multiple criteria alternatives with partial information and use of ordinal and cardinal strength of preferences, IEEE Trans. Syst., Man. Cyb.: Part A 30 (2000) 787-801.

[11] A. Mateos, A. Jiménez, S. Ríos Insua, Monte Carlo simulation techniques for group decision-making with incomplete information, Euro. J. Oper. Res. 174 (2006) 1842-1864.

[12] K. Park, Mathematical programming models for characterizing dominance and potential optimality when multicriteria alternative values and weights are simultaneously incomplete, IEEE Trans. Syst., Man. Cyb.: Part A 34 (2004) 601614

[13] J. Puerto, A.M. Marmol, L. Monroy, F.R. Fernăndez, Decision criteria with partial information, IEEE Trans. Oper. Res. 7 (2000) 51-65.

[14] H. Raiffa, The Art and Science of Negotiation, Harvard University Press, Cambridge, 1982.

[15] D. Ríos Insua, Sensitivity Analysis in Multi-objective Decision Making, Springer, New York, 1990

[16] D. Ríos, S. French, A framework for sensitivity analysis in discrete multiobjective decision-making, Euro. J. Oper. Res. 54 (1991) 176-190.

[17] A. Sage, C.C. White, Ariadne: a knowledge-based interactive system for planning and decision support, IEEE Trans. Syst., Man. Cyb.: Part A 14 (1984) $35-47$

[18] A. Salo, R.P. Hämäläinen, Preference ratio in multiattribute evaluation (PRIME) - elicitation and decision procedures under incomplete information, IEEE Trans. Syst., Man. Cyb.: Part A 31 (2001) 533-545.

[19] P. Sarabando, L.C. Dias, Multi-attribute choice with ordinal information: a comparison of different decision rules, IEEE Trans. Syst., Man. Cyb.: Part A 39 (2009) 545-554.

[20] P. Sarabando, L.C. Dias, Simple procedures of choice in multicriteria problems without precise information about the alternatives values, Comput. Oper. Res. 37 (2010) 2239-2247.

[21] T.J. Stewart, Robustness of additive value function method in MCDM, J. MultiCrit. Decis. Anal. 5 (1996) 301-309.

[22] W.G. Stillwell, D.A. Seaver, W.A. Edwards, Comparison of weight approximation techniques in multiattribute utility decision making, Org. Behav. Human Decis. Proc. 28 (1981) 62-77.

[23] M. Weber, Decision making with incomplete information, Euro. J. Oper. Res. 28 (1987) 44-57.

[24] R.L. Winkler, W.L. Hays, Statistics: Probability, Inference and Decision, Holt Rinehart \& Winston, New York, 1985.

[25] Z. Xu, A method for multiple attribute decision making with incomplete weight information in linguistic setting, Knowl.-Based Syst. 20 (2007) 719725.

[26] Y.Xu, Q. Da, A method for multiple attribute decision making with incomplete weight information under uncertain linguistic environment, Knowl.-Based Syst. 21 (2008) 837-841.

[27] Z. Yue, An extended TOPSIS for determining weights of decision makers with interval numbers, Knowl.-Based Syst. 24 (2011) 146-153. 